\section{The Whole Genome Sequence of Fusarium redolens Strain YP04, a Pathogen that Causes Root Rot of American Ginseng}

\author{
Sanhong Fan, ${ }^{1}$ Qiang Wang, ${ }^{2}$ Jichen Dai, ${ }^{2}$ Jinglong Jiang, ${ }^{3}$ Xiaoping $\mathrm{Hu},{ }^{2, \dagger}$ and \\ Krishna V. Subbarao ${ }^{4}$ \\ ${ }^{1}$ College of Life Science, Northwest A\&F University, Yangling, Shaanxi 712100, China \\ ${ }^{2}$ State Key Laboratory of Crop Stress Biology for Arid Areas and College of Plant Protection, Northwest \\ A\&F University, Yangling, Shaanxi 712100, China \\ ${ }^{3}$ Shaanxi Key Laboratory of Resource Biology, School of Bioscience and Engineering, Shaanxi \\ University of Technology, Hanzhong, Shaanxi 723001, China \\ ${ }^{4}$ Department of Plant Pathology, University of California, Davis, CA 93905, U.S.A.
}

\begin{abstract}
Fusarium redolens was previously reported as a plant pathogen or an endophyte that is closely related to $F$. oxysporum, a notoriously significant soilborne phytopathogen. Subsequent studies demonstrated the unique nature of $F$. redolens, which was considered a distinct species that causes multiple symptoms on multiple hosts. It was recently identified as a pathogen that causes root rot of American ginseng. Currently, few high-quality $F$. redolens genome sequences exist in the public database. Here, we report the whole-genome sequence of $F$. redolens strain YP04, based on a hybrid assembly of long- and short-read sequencing with PacBio and Illumina platforms, respectively. The assembly consists of 40 configs with a total length of $52.8 \mathrm{Mb}$ nuclear genomic DNA and $49.6 \mathrm{~kb}$ complete mitochondrial genomic DNA, and encodes a total of 18,985 genes, including 18,517 protein-coding genes and 469 RNA genes which were functionally annotated. In total, 4,606 proteins were identified in the pathogen-host interactions database, suggesting that they were likely involved in pathogenicity and host-pathogen interactions, while 41 secondary metabolite synthesis clusters were predicted and annotated. This is the first high-quality whole genome of $F$. redolens, providing an important community resource for genome evolution, host-pathogen interaction, and secondary metabolite biosynthesis studies.
\end{abstract}

Fusarium redolens was identified as a member of Fusarium spp. by Wollenweber as early as 1913. The taxonomic position of $F$. redolens has been controversial for a long time, with several publications showing $F$. redolens as a member of the $F$. oxysporum complex or a variety of $F$. oxysporum (Gordon 1952; Nelson et al. 1983; Snyder and Hansen 1940), because distinguishing species through morphological characteristics is difficult. At the end of the 20th century, evidence from DNA sequences revealed that $F$. redolens and $F$. oxysporum were separate species and that $F$. hostae could be classified as a species closer to F. redolens (Baayen et al. 2001; Waalwijk et al. 1996).

F. redolens causes various symptoms, including wilt, seedling damping-off, and root rots. The pathogenic $F$. redolens was first isolated from Pisum sativum with symptoms of wilt and foot rot (Wollenweber 1913). Subsequent studies found that it was associated with damping-off in Aleppo pine (Baayen and Gams 1988); Fusarium yellows in chickpea (Jiménez-Fernández et al. 2011); and root rot in soybean (Bienapfl et al. 2010), asparagus (Borrego-Benjumea et al. 2014), Panax ginseng (Guan et al. 2014), and American ginseng (Fan et al. in press). In addition, F. redolens as an endophyte is capable of producing several bioactive compounds. Examples include an

\footnotetext{
${ }^{\dagger}$ Corresponding author: X. Hu; xphu@nwsuaf.edu.cn

*The $e$-Xtra logo stands for "electronic extra" and indicates that supplementary tables are published
} online.

The author(s) declare no conflict of interest.

Accepted for publication 3 May 2021.

\section{Funding}

This work was supported by the Integrated Extension Project of Agricultural Science and Technology Innovation in Shaanxi Province (NYKJ-2019-YL27), the Special Project of Technological Innovation Guidance in Shaanxi Province (2020QFY-06), and the Open Fund from Shaanxi Key Laboratory of Resource Biology, Shaanxi Province, P. R. China (SLGPT2019KF04-03).

\section{Keywords}

American ginseng, root rot, Fusarium redolens, host-pathogen interaction, secondary metabolites, whole genome sequence 
Table 1. Genome statistics of Fusarium redolens strain YP04

\begin{tabular}{lc} 
Statistics & F. redolens strain YP04 \\
Assembly & \\
Depth of PacBio Sequel reads & $587 \times$ \\
Depth of DNBSEQ pair-end reads & $110 \times$ \\
Number of contigs & $52,841,013 \mathrm{bp}$ \\
Total length of DNA & $49,602 \mathrm{bp}$ \\
Total length of mitochondrial DNA & $4,335,199 \mathrm{bp}$ \\
Contig N So $_{\text {Size of top } 13 \text { contigs }}$ & $47,685,092 \mathrm{bp}$ \\
GC content & $46.36 \%$ \\
BUSCO completeness & $99.80 \%$ \\
Repeat rate & $13.48 \%$ \\
Annotation & \\
Total genes & 18,986 \\
Annotated transfer RNA genes & $306(8 \mathrm{pseudogenes)}$ \\
Annotated ribosomal RNA genes & 112 \\
Annotated other RNA genes & 51 \\
Annotated protein coding genes & 18,517 \\
Secreted proteins & 1,170 \\
Carbohydrate-active enzymes (CAZymes) & 652 \\
Secreted CAZymes & 351 \\
Pathogen-host interaction proteins & 4,606 \\
Secondary metabolite biosynthesis clusters & 41 \\
\hline
\end{tabular}

endophytic $F$. redolens strain isolated from Himalayan yew producing anticancer compound, taxol (Garyali et al. 2013); the endophytic F. redolens Dzf2, from the rhizome of Dioscorea zingiberensis, engineered to produce beauvericin, a pesticide (Xu et al. 2011); and F. redolens 6WBY3 from Fritillaria unibracteata var. wabuensis that produces steroidal alkaloids, peimisine, and imperialine-3 $\beta$-D-glucoside (Pan et al. 2015).

American ginseng is an important herbal medicine and an economically significant crop in the world. Although American ginseng is native to the United States and Canada, China has become the third-largest producer and the largest consumer of American ginseng with the increasing market demand. Continuous cropping has led to serious problems in commercial production of American ginseng due to rapid accumulation of root pathogens and associated disease incidences (Zhang etal. 2020). YP04 is a representative strain of Fusarium redolens isolated and identified from rotten roots of American ginseng in Liuba County, Shaanxi Province, China (Fan et al. in press). Currently, few high-quality $F$. redolens genomes are publicly available, which limits the study of genome evolution and host-pathogen interactions in this fungal pathogen. We assembled a high-quality genome for the $F$. redolens strain YP04 by combining PacBio and Illumina sequencing datasets. This genome resource can facilitate research on host-F. redolens interactions, and mining of novel secondary metabolites genes or gene clusters for potential commercial exploitation.

\section{Genome Sequencing and Assembly}

The F. redolens strain YP04 was cultured in potato dextrose broth media on a rotary shaker at $160 \mathrm{rpm}$ and $25^{\circ} \mathrm{C}$ for 3 days in the dark, and mycelia were collected for total genomic DNA extraction with a conventional cetyltrimethylammonium bromide method (Möller et al. 1992). The genomic DNA was sent to Beijing Genome Institute (BGI, Wuhan, China) for sequencing using both PacBio and Illumina platforms. The $>10-k b$ DNA fragments were utilized for the construction of a long insert library and sequenced using Sequel Sequencing Kit 2.0 (Pacific Biosciences), while the genomic DNA was disrupted into approximately 300- to 400-bp DNA fragments for constructing a short insert library and sequenced to produce paired-end reads of $150 \mathrm{bp}$ on a DNBSEQ platform (BGI). After removing low-quality (length $<1,000 \mathrm{bp}$ ) and adapter contamination reads, in total, 31.0 Gb (approximately 587x coverage) PacBio Sequel long reads and $5.8 \mathrm{~Gb}$ (approximately 110x coverage) paired-end short reads were obtained (Table 1) and used for de novo genome assembly with Canu v2.1.1 (corrected error rate parameter set at 0.025) (Koren et al. 2017). The contigs were further polished with raw PacBio reads by Gcpp v1.9, and short paired-end reads by Freebayes v1.3.2 (Garrison and Marth 2012). The final assembly consisted of 40 contigs with a total length of 52,791,411 bp of nuclear genomic DNA, a 4,335,199-bp $\mathrm{N}_{50}$ contig, and a 49,602-bp complete mitochondrial genomic DNA sequence. The completeness of the genome assembly was estimated 
Table 2. Predicted secondary metabolite biosynthesis clusters in Fusarium redolens strain YP04 ${ }^{\mathrm{a}}$

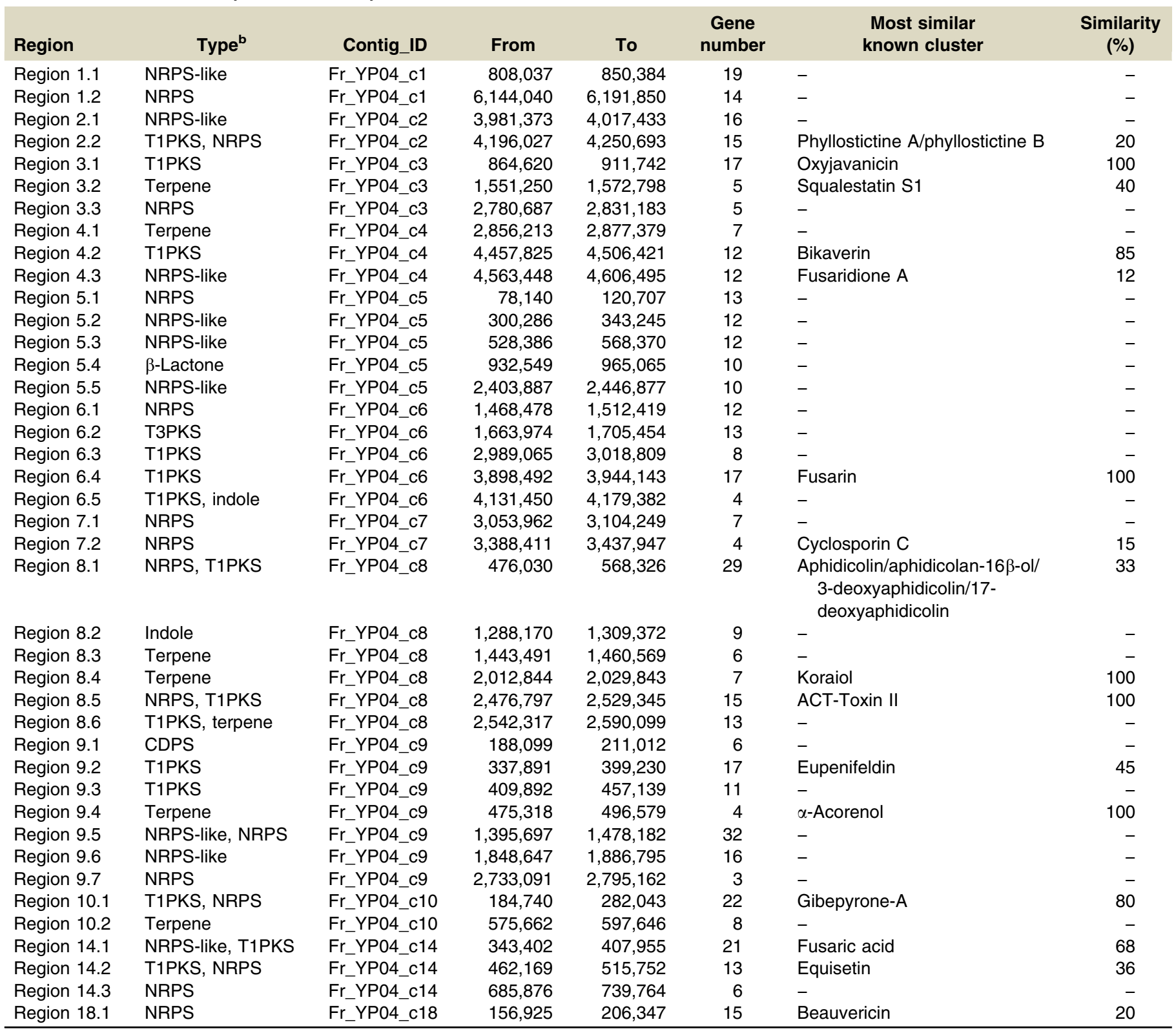

a Using strictness 'relaxed'.

${ }^{\mathrm{b}}$ NRPS $=$ nonribosomal peptide synthetase, T1PKS = type I polyketide synthase, and CDPS = cyclodipeptide synthase.

by BUSCO v5.1.2 based on the lineage-specific profile libraries, including eukaryota_odb10, fungi_odb10, ascomycetes_odb10, sordariomycetes_odb10, and hypocreales_odb10 (Simão et al. 2015), in which 252 of 255 (98.9\%), 758 of 758 (100.0\%), 1,701 of 1,706 (99.7\%), 3,811 of $3,817(99.8 \%)$, and 4,484 of $4,494(99.8 \%)$ BUSCO groups, respectively, were identified in the genome of $F$. redolens strain YP04. In all, $13.48 \%$ of the genome sequence was annotated as repetitive sequences with REPET package v3.0 (Flutre et al. 2011) and the telomeric repeat sequence of TTAGGG (van Dam et al. 2017) enriched on both ends of 3 contigs and the $5^{\prime}$ or $3^{\prime}$ end of 18 contigs. The genome assembly comparison between F. redolens strain YP04 and reference strains of four Fusarium spp. (F. oxysporum, F. solani, F. verticillioides, and F. graminearum) (Supplementary Table S1) indicated that the F. redolens strain YP04 genome had a high-quality assembly with contigs at nearly chromosome-arm level.

\section{Genome Annotation}

Prediction and evaluation of protein coding genes were conducted using a previously described pipeline, FunGAP Pipeline v1.1 (Min et al. 2017). The 23.2-Gb paired-ends RNAsequencing reads of $F$. redolens strain A4 (BioProject PRJNA444683 and Sequence Read 
Archive SRR7495239, submitted by DOE Joint Genome Institute in 2008) was downloaded and provided to the pipeline, which allowed for high-quality gene calling. To improve the accuracy of ab initio prediction, the gene model was optimized by using a well-annotated genome of $F$. oxysporum f. sp. lycopersici 4287 (ASM14995v2). The sister proteomes were generated by merging the proteome sequences of $F$. oxysporum (ASM14995v2 and GCA_000260075.2), F. verticillioides (ASM14955v1), and F. fujikuroi (IMI58289V2), contributing to evaluations of predicted gene models. RepeatMasker v4.0.9 was employed for identifying repeat sequences; HISAT v2.2.1 (Kim et al. 2015) and Trinity v2.11.0 (Grabherr et al. 2011) were used for messenger RNA assembly; and Augustus v3.3.3 (Stanke et al. 2008), Braker v2.1.5 (Hoff et al. 2019), and Maker v2.31.10 based on GeneMark-ES v 4.61 (Ter-Hovhannisyan et al. 2008) were used for gene prediction. In total, 18,517 protein-coding genes were predicted in the genome of $F$. redolens strain YP04 with an average coding sequence length of 1,329 bp. Each protein was functionally annotated using the BLASTp in Swissport (pident > 30\%, e-value < 1e-7) and NCBI nr database (pident > $50 \%$, e-value $<1 e-7$ ), and protein domains were scanned against the Pfam $\vee 32.0$ database with Hmmer v3.3 (Eddy 2011). Noncoding RNAs, including 306 transfer RNAs (tRNAs), 112 ribosomal RNAs, and 51 other RNAs were predicted by tRNAscan-SE v2.0 (Lowe and Chan 2016) and Cmscan v1.3.2 in the Rfam14.4 (Kalvari et al. 2021).

To identify the potential pathogen-plant interaction proteins, BLASTp (pident $>30 \%$, e-value $<1 \mathrm{e}-7$ ) was performed against the pathogen-host interaction base 4.9 (Urban et al. 2020) and, in total, 4,606 proteins, including 221 increased virulence, 2,108 reduced virulence, 291 loss of pathogenicity, 176 lethal, 35 effector, and 19 potential chemistry target proteins, were detected (Supplementary Table S2). In total, 1,170 secreted proteins were predicted in the genome of the strain YP04 using SingalP 5.0 (Almagro Armenteros et al. 2019), TMHMM v 2.0, and WoLF PSORT (Hortona et al. 2006). Because the carbohydrate-active enzymes (CAZymes), especially secretory CAZymes, play crucial roles during fungal infection, we annotated all the CAZymes in the YP04 genome using local Run-dbcan v2.0.11 based on dbCAN2 database V9 (supported by $\geq 2$ tools) (Zhang et al. 2018). In total, 652 and 320 CAZymes and secretory CAZymes, respectively, were identified in F. redolens strain YP04 (Supplementary Table S3), which included 359 and 191 glycoside hydrolases (GHs) and secretory GHs, 119 and 2 glycosyl transferases (GTs) and secretory GTs, 90 and 59 auxiliary activities (Aas) and secretory Aas, 47 and 35 carbohydrate esterases (CEs) and secretory CEs, 26 and 24 polysaccharide lyases (PLs) and secretory PLs, and 56 and 38 carbohydrate-binding modules (CBMs) and secretory CBMs.

Secondary metabolites, especially mycotoxins, are involved in pathogen-host and pathogen-environment interactions (Ma et al. 2013). In total, 41 gene clusters (Table 2) involved in the biosynthesis of secondary metabolites (17 nonribosomal peptide synthetase [NRPS], 9 type I polyketide synthase-NRPS, 7 terpene, and 1 indole) in the $F$. redolens strain YP04 genome were predicted using the online tool antiSMASH v5.22 (Blin et al. 2019). Five clusters shared a 100\% similarity with several well-studied clusters, including fusarin, oxyjavanicin, $\alpha$-acorenol, and koraiol biosynthesis gene clusters from F. fujikuroi (BGC0001268, BGC0001242, BGC0001481, and BGC000164) and an ACT-Toxin II cluster from A. alternata (BGC0001254). Three clusters had a $>50 \%$ similarity with known clusters, including bikaverin and gibepyrone-A clusters from $F$. fujikuroi (BGC0000030 and BGC0001606) and a fusaric acid cluster from F. verticilioides (BGC0001190). In addition, gene clusters likely involved in the synthesis of squalestatin S1, phyllostictine, fusaridione $\mathrm{A}$, cyclosporin $\mathrm{C}$, aphidicolin, eupenifeldin, equisetin, and beauvericin in the genome of $F$. redolens strain YP04 require further evaluation. Hence, the strain will be useful for investigating biosynthesis of potential bioactive compounds such as mycotoxins, antimicrobial agents, insecticides, or drugs.

In this study, we provided the first high-quality genome sequence of $F$. redolens strain YP04 based on the high-coverage sequencing data generated by the PacBio Sequel and DNBSEQ platform. The genome represents a useful resource for future research related to the evolution of $F u$ sarium spp., interactions between F. redolens and host, and biosynthesis of secondary metabolites.

\section{Data Availability}

The Whole Genome Shotgun project of $F$. redolens strain YP04 has been deposited at DNA

Data Bank of Japan/European Nucleotide Archive/GenBank under accession number JAFFJM000000000.1 (BioProject PRJNA692049, BioSample SAMN17312464, and Assembly GCA_017562085.1). 


\section{Literature Cited}

Almagro Armenteros, J. J. A., Tsirigos, K. D., Sonderby, C. K., Petersen, T. N., Winther, O., Brunak, S., von Heijne, G., and Nielsen, H. 2019. SignalP 5.0 improves signal peptide predictions using deep neural networks. Nat. Biotechnol. 37:420-423.

Baayen, R. P., and Gams, W. 1988. The Elegans fusaria causing wilt disease of carnation. I. Taxonomy. Neth. J. Plant Pathol. 94:273-288.

Baayen, R. P., O'Donnell, K., Breeuwsma, S., Geiser, D. M., and Waalwijk, C. 2001. Molecular relationships of fungi within the Fusarium redolens- $F$. hostae clade. Phytopathology 91:1037-1044.

Bienapfl, J. C., Malvick, D. K., and Percich, J. A. 2010. First report of Fusarium redolens causing root rot of soybean in Minnesota. Plant Dis. 94:1069.

Blin, K., Shaw, S., Steinke, K., Villebro, R., Ziemert, N., Lee, S. Y., Medema, M. H., and Weber, T. 2019. antiSMASH 5.0: Updates to the secondary metabolite genome mining pipeline. Nucleic Acids Res. 47:W81-W87.

Borrego-Benjumea, A., Basallote-Ureba, M. J., Melero-Vara, J. M., and Abbasi, P. A. 2014. Characterization of Fusarium isolates from asparagus fields in southwestern Ontario and influence of soil organic amendments on Fusarium crown and root rot. Phytopathology 104:403-415.

Eddy, S. R. 2011. Accelerated profile HMM searches. PLOS Comput. Biol. 7: e1002195.

Fan, S. H., Zhao, F. J., Zhang, J. G., Shang, W. J., and Hu, X. P. American ginseng root rot caused by Fusarium redolens in China. 2021. Plant Dis. In press.

Flutre, T., Duprat, E., Feuillet, C., and Quesneville, H. 2011. Considering transposable element diversification in de novo annotation approaches. PLoS One 6:e16526.

Garrison, E., and Marth, G. 2012. Haplotype-based variant detection from short-read sequencing. arXiv 1207.3907v2 [q-bio.GN].

Garyali, S., Kumar, A., and Reddy, M. S. 2013. Taxol production by an endophytic fungus, Fusarium redolens, isolated from Himalayan yew. J. Microbiol. Biotechnol. 23: 1372-1380.

Gordon, W. L. 1952. The occurrence of Fusarium species in Canada. II. Prevalence and taxonomy of Fusarium species in cereal seed. Can. J. Bot. 30:209-251.

Grabherr, M. G., Haas, B. J., Yassour, M., Levin, J. Z., Thompson, D. A., Amit, I., Adiconis, X., Fan, L., Raychowdhury, R., Zeng, Q. D., Chen, Z. H., Mauceli, E., Hacohen, N., Gnirke, A., Rhind, N., di Palma, F., Birren, B. W., Nusbaum, C., Lindblad-Toh, K., Friedman, N., and Regev, A. 2011. Full-length transcriptome assembly from RNA-Seq data without a reference genome. Nat. Biotechnol. 29: 644-652.

Guan, Y. M., Lu, B. H., Wang, Y., Gao, J., and Wu, L. J. 2014. First report of root rot caused by Fusarium redolens on ginseng (Panax ginseng) in Jilin Province of China. Plant Dis. 98:844.

Hoff, K. J., Lomsadze, A., Borodovsky, M., and Stanke, M. 2019. Whole-genome annotation with BRAKER. Methods Mol. Biol. 1962:65-95.

Hortona, P., Park, K. J., Obayashi, T., and Nakai, K. 2006. Protein subcellular localization prediction with WoLF PSORT. Pages 39-48 in: Proc. 4th Asia-Pac. Bioinf. Conf. Series on Advances in Bioinformatics and Computational Biology.

Jiménez-Fernández, D., Navas-Cortés, J. A., Montes-Borrego, M., Jiménez-Díaz, R. M., and Landa, B. B. 2011. Molecular and pathogenic characterization of Fusarium redolens, a new causal agent of Fusarium yellows in chickpea. Plant Dis. 95:860-870.

Kalvari, I., Nawrocki, E. P., Ontiveros-Palacios, N., Argasinska, J., Lamkiewicz, K., Marz, M., Griffiths-Jones, S., Toffano-Nioche, C., Gautheret, D., Weinberg, Z., Rivas, E., Eddy, S. R., Finn, R. D., Bateman, A., and Petrov, A. I. 2021.
Rfam 14: Expanded coverage of metagenomic, viral and microRNA families. Nucleic Acids Res. 49:D192-D200.

Kim, D., Langmead, B., and Salzberg, S. L. 2015. HISAT: A fast spliced aligner with low memory requirements. Nat. Methods 12:357-360.

Koren, S., Walenz, B. P., Berlin, K., Miller, J. R., Bergman, N. H., and Phillippy, A. M. 2017. Canu: Scalable and accurate long-read assembly via adaptive k-mer weighting and repeat separation. Genome Res. 27:722-736.

Lowe, T. M., and Chan, P. P. 2016. tRNAscan-SE On-line: Integrating search and context for analysis of transfer RNA genes. Nucleic Acids Res. 44:W54-W57.

Ma, L. J., Geiser, D. M., Proctor, R. H., Rooney, A. P., O'Donnell, K., Trail, F., Gardiner, D. M., Manners, J. M., and Kazan, K. 2013. Fusarium pathogenomics. Annu. Rev. Microbiol. 67:399-416.

Min, B., Grigoriev, I. V., and Choi, I. G. 2017. FunGAP: Fungal genome annotation pipeline using evidence-based gene model evaluation. Bioinformatics 33:2936-2937.

Möller, E. M., Bahnweg, G., Sandermann, H., and Geiger, H. H. 1992. A simple and efficient protocol for isolation of high molecular weight DNA from filamentous fungi, fruit bodies, and infected plant tissues. Nucleic Acids Res. 20:6115-6116.

Nelson, P. E., Toussoun, T. A., and Marasas, W. F. O. 1983. Fusarium species. An Illustrated Manual for Identification. Pennsylvania State University Press, University Park, PA, U.S.A.

Pan, B. F., Su, X., Hu, B., Yang, N., Chen, Q., and Wu, W. 2015. Fusarium redolens $6 \mathrm{WBY} 3$, an endophytic fungus isolated from Fritillaria unibracteata var. wabuensis, produces peimisine and imperialine-3beta-D-glucoside. Fitoterapia 103:213-221.

Simão, F. A., Waterhouse, R. M., Ioannidis, P., Kriventseva, E. V., and Zdobnov, E. M. 2015. BUSCO: Assessing genome assembly and annotation completeness with single-copy orthologs. Bioinformatics 31:3210-3212.

Snyder, W. C., and Hansen, H. N. 1940. The species concept in Fusarium. Am. J. Bot. 27:64-67.

Stanke, M., Diekhans, M., Baertsch, R., and Haussler, D. 2008. Using native and syntenically mapped cDNA alignments to improve de novo gene finding. Bioinformatics 24:637-644.

Ter-Hovhannisyan, V., Lomsadze, A., Chernoff, Y. O., and Borodovsky, M. 2008. Gene prediction in novel fungal genomes using an ab initio algorithm with unsupervised training. Genome Res. 18:1979-1990.

Urban, M., Cuzick, A., Seager, J., Wood, V., Rutherford, K., Venkatesh, S. Y., De Silva, N., Martinez, M. C., Pedro, H., Yates, A. D., Hassani-Pak, K., and Hammond-Kosack, K. E. 2020. PHI-base: The pathogen-host interactions database. Nucleic Acids Res. 48:D613-D620.

van Dam, P., Fokkens, L., Ayukawa, Y., van der Gragt, M., Ter Horst, A., Brankovics, B., Houterman, P. M., Arie, T., and Rep, M. 2017. A mobile pathogenicity chromosome in Fusarium oxysporum for infection of multiple cucurbit species. Sci. Rep. 7:9042.

Waalwijk, C., de Koning, J. R. A., Baayen, R. P., and Gams, W. 1996. Discordant groupings of Fusarium spp. from sections Elegans, Liseola and Dlaminia based on ribosomal ITS1 and ITS2 sequences. Mycologia 88:361-368.

Wollenweber, H. W. 1913. Studies on the Fusarium problem. Phytopathology 3:26.

Xu, L. J., Liu, Y. S., Zhou, L. G., and Wu, J. Y. 2011. Modeling of Fusarium redolens Dzf2 mycelial growth kinetics and optimal fed-batch fermentation for beauvericin production. J. Ind. Microbiol. Biotechnol. 38:1187-1192.

Zhang, H., Yohe, T., Huang, L., Entwistle, S., Wu, P., Yang, Z., Busk, P. K., Xu, Y., and Yin, Y. 2018. dbCAN2: A meta server for automated carbohydrate-active enzyme annotation. Nucleic Acids Res. 46:W95-W101.

Zhang, J., Fan, S., Qin, J., Dai, J., Zhao, F., Gao, L., Lian, X., Shang, W., Xu, X., and $\mathrm{Hu}, X .2020$. Changes in the microbiome in the soil of an American ginseng continuous plantation. Front. Plant Sci. 11:572199. 